\title{
Survey of the Porcine Trichinellosis in Slaughtered Pigs in Nay Pyi Taw, Myanmar
}

\author{
Tin Aye Khaing ${ }^{1,2, a^{*}}$, Saw Bawm ${ }^{2, b}$, Kyaw San Linn ${ }^{2, c}$, Tin Tin Myaing ${ }^{3, d}$, \\ Lat Lat Htun ${ }^{2, e}$ \\ ${ }^{1}$ Department of Veterinary and Slaughterhouses, Nay Pyi Taw Development Committee, \\ Nay Pyi Taw 15011, Myanmar \\ ${ }^{2}$ Department of Pharmacology and Parasitology, University of Veterinary Science Yezin, \\ Nay Pyi Taw 15013, Myanmar \\ ${ }^{3}$ Pro-Rector (Academic) (retired), University of Veterinary Science, Yezin, Nay Pyi Taw, Myanmar \\ a tinayekhaing007@gmail.com, b bestshadow@gmail.com, \\ c serroundvettinaye996789@gmail.com, d dr.tintinmyaing@gmail.com, ${ }^{e}$ m.latht@gmail.com.
}

Keywords: slaughterhouse; trichinellosis; prevalence; risk factor; Nay Pyi Taw

\begin{abstract}
A cross-sectional study was conducted to determine the prevalence and associated risk factors of trichinellosis in slaughtered pigs from three slaughterhouses located in Pyinmana, Lewe and Tatkon Townships, Nay Pyi Taw, Myanmar. A total of 270 muscle samples (tongue, masseter and diaphragmatic muscles from each pig) from 90 slaughtered pigs were randomly collected and examined for the presence of Trichinella larvae by using artificial digestion method. Three pigs (3.3\%) showed Trichinella positive and larval intensity per gram (lpg) was 0.2. For the determination of associated risk factors of trichinellosis, questionnaire interviews to officials of slaughterhouses and owners of slaughtered pigs were conducted. The presence of rodents around the pig farm and type of feed (use of uncooked waste food) were identified as associated risk factors for trichinellosis. Porcine trichinellosis occurred with a low rate of infection in this study.
\end{abstract}

\section{Introduction}

Humans suffer from numerous parasitic foodborne zoonoses and many of which are caused by helminths [1]. One of the foodborne zoonotic diseases is trichinellosis and is caused by a tissuedwelling nematode of the genus Trichinella. WHO reported that some swine herds in some of developed as well as developing countries had a $50 \%$ prevalence of trichinellosis, and thousands of human cases had been documented [2]. Trichinellosis occurs globally and has commonly been reported in Southeast Asia. Approximately 11 million people worldwide are at risk of infection [3].

Trichinella parasite is normally associated with pigs and dogs. Rats, cats and certain wild carnivores are also capable of acting as zoonotic reservoirs [4]. All mammalian species are susceptible to experimental infection with Trichinella and naturally infected species include humans, carnivorous animals (cats, dogs, wolves, foxes, bears, walruses and seals), omnivores (pigs, wild pigs), rodents (rats, mice and marmots), and herbivores (horses, sheep, coypu and rabbits) [5].

Clinical manifestations of human trichinellosis are gastrointestinal symptoms, facial oedema, diffuse myalgia, conjunctivitis, fever, headache, malaise and in severe infection may be dead. The most frequent cause of death is myocarditis as a result of invasion of cardiac muscle and heart failure $[6,7]$.

Although there are many reports on prevalence of trichinellosis from many countries including neighboring countries of Myanmar such as China, India, Laos, Thailand, Indonesia and Malaysia, a little information was reported in Myanmar. In 1989 and 1990, Central Veterinary Laboratory in Yangon, Myanmar reported that only one sample out of 635 samples $(0.16 \%)$ had Trichinella larvae in pooled sample from diaphragmatic muscle, tongue muscle, masseter muscle and intercostal muscles. In 2006, Maw et al. [8] investigated that the prevalence of trichinellosis was only $1.13 \%$ in slaughterhouse of Insein Township, Yangon, Myanmar. 
In Myanmar, most of pig rearing system is free-ranging or backyard farm system and some are tethered by smallholder farmers. Most of pig farmers use uncooked kitchen waste and swill feeding which may contain infected scraps. Therefore, there is favourable for synanthropic or domestic cycle of Trichinella.

Myanmar traditional eating habit is based on well-cooking, but due to introduction of new food style such as barbecue and dishes based on raw or undercooked pork or pork product may lead to trichinellosis outbreaks. Many countries of the world are trying to control zoonoses in possible means. Without investigation on factors affecting the trichinellosis in domestic pigs, there would be difficult to control trichinellosis as well as in conducting public education programme. Although there are many favourable conditions in Myanmar, there is no reliable data of porcine trichinellosis. Therefore, study on the prevalence and associated risk factors of trichinellosis was carried out in slaughtered pigs from slaughterhouses located in Pyinmana, Lewe and Tatkon Townships, Nay Pyi Taw, Myanmar.

\section{Materials and methods}

\subsection{Study design, study area, study sample, study period and sample size}

A cross-sectional study was conducted. Sample collection was performed in three slaughterhouses located in Pyinmana, Lewe and Tatkon Townships, Nay Pyi Taw, Myanmar. It is located between latitude $19^{\circ} 45^{\prime} \mathrm{N}$ and longitude $96^{\circ} 6^{\prime} \mathrm{E}$ and with climate data; the altitude is $115 \mathrm{~m}$ above sea level, annual rainfall is $115 \mathrm{~mm}$, and annual temperature is $21.2-32^{\circ} \mathrm{C}$. The targeted population was 180,000 pigs in three townships [11] during the sampling period. A total of 270 muscles (tongue, masseter and diaphragmatic muscles from each pig) from 90 slaughtered pigs (30 pigs from each slaughterhouse) were collected and examined during January to March 2012. The number of sampled pigs was calculated using the formula stated by Thrusfield [9]. An expected prevalence of $10 \%$ with a confidence level of $95 \%$ was used in this unit.

\subsection{Sample collection}

Each 100 grams of tongue, masseter, and diaphragmatic muscles from each pig were collected. Sampling from pigs was randomly conducted just after slaughter at slaughterhouses. Twenty four muscle samples from 8 pigs were collected weekly during the study period.

\subsection{Questionnaire study}

Questionnaires including source (owner's name and address), age, sex, breed, body weight of slaughtered pig were developed from officials of slaughterhouses. Questionnaires for rearing system, feed type, presence or absence of rodents around the pig farm, use of anthelmintic and occurrence of tail biting in pigs were also developed and collected from the owners of slaughtered pigs.

\subsection{Artificial digestion method for Trichinella larvae}

\subsubsection{Preparation of muscle samples and digestion fluid}

Fat, perymisium, superficial layer of the tongue and parts of the tendon present in the collected samples were removed as much as possible. It needed to be careful not to remove muscle tissues, especially those near the tendon insertion. After removing these matters, about 50 grams of muscle tissue were cut into very small pieces (as small as possible) and were blended. Five grams of these blended muscle was used for artificial digestion.

In order to prepare digestion fluid, $0.8 \mathrm{ml}$ of $\mathrm{HCl}(25 \%)$ was added in a beaker containing $100 \mathrm{ml}$ of distilled water and $0.5 \mathrm{ml}$ of pepsin $(1: 10,000)$ (Wako Company, Japan) and mixed thoroughly. 


\subsubsection{Artificial digestion method}

Five grams of blended muscle sample were put in a digestion fluid beaker and dispersed with a spatula. A magnetic stirring rod was put and covered with aluminum foil and then the beaker was placed onto a preheated plate of magnetic stirrer. The magnetic stirrer was adjusted to maintain constant temperature of $44-46^{\circ} \mathrm{C}$ throughout the operation and checked the temperature every five minutes. The stirring process was continued until the meat particles disappeared for $30 \mathrm{~min}$. The stirrer was then switched off and the digested fluid was poured through the sieve $(250 \mu \mathrm{m})$ into a steel bowl.

The sieve was gently but thoroughly spray washed with $20 \mathrm{ml}$ of warm tap water and added to sieved digested fluid. About $120 \mathrm{ml}$ of digested fluid was transferred to a Baermann funnel and then kept in standing position for 30 minutes. After that, $12 \mathrm{ml}$ of sediment of digested fluid was quickly run off into the measuring cylinder. It was continued to stand for $10 \mathrm{~min}$ and then $9 \mathrm{ml}$ of supernatant fluid was withdrawn. The remaining $3 \mathrm{ml}$ of sediment was poured into Petri dish and examined under a dissecting microscope. If the suspension was still not sufficiently clear, it was washed with water till 4 times.

\subsubsection{Identification of Trichinella larvae}

Three milliliters of suspension were divided and put into 5 Petri dishes and examined under a dissecting microscope. When suspected larva was observed, it was examined under a stereo microscope (A.KRUSS Optronic GmbH, Germany) at $400 \times$ magnification.

\subsection{Statistical analysis}

The relationship between Trichinella infection and hypothesized risk variables such as age, gender, body weight, rearing system, feed type, presence or absence of rodents around the farm and usage of anthelmintics were examined by testing its significance using the Pearson Chi-square test at $\alpha=0.05$.

\section{Results}

\subsection{Prevalence of trichinellosis in slaughtered pigs}

Of 270 muscle samples from 90 slaughtered pigs examined, 3 samples from 3 pigs $(3.3 \%)$ were observed as Trichinella positive by artificial digestion method (Plate 1). The 3 positive muscles were 2 diaphragmatic muscle and 1 masseter muscle. Those positive muscle samples were from pigs of Lewe Township.

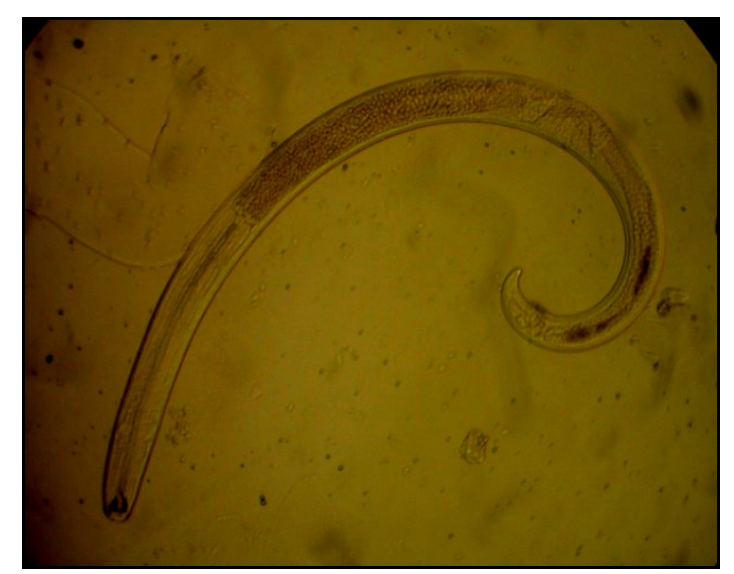

Plate 1. Appearance of Trichinella larva under stereo microscope ((A.KRUSS Optronic GmbH, Germany) (400 x magnification) 
The distribution and odds ratio of associated risk factors concerning porcine trichinellosis is shown in Table 1.

Table 1. Distribution and odds ratio of associated risk factors concerning porcine trichinellosis in Nay Pyi Taw, Myanmar

\begin{tabular}{|l|l|l|l|l|l|l|l|}
\hline Factor & Level & $\mathrm{n}$ & $\begin{array}{l}\text { Positive } \\
\text { case }\end{array}$ & $\begin{array}{l}\text { Negative } \\
\text { case }\end{array}$ & Odds ratio & $\begin{array}{l}95 \% \\
\text { Confidence } \\
\text { interval }\end{array}$ & $\mathrm{p}$ value \\
\hline Age & $\geq 8$ months & 26 & 0 & 26 & 0.071 & $0.946-$ & 0.554 \\
& $<8$ months & 64 & 3 & 61 & & 1.009 & \\
\hline Gender & Male & 56 & 2 & 54 & 0.026 & $0.71-9.38$ & 1.000 \\
& Female & 34 & 1 & 33 & & & \\
\hline Body weight & $<40$ viss & 17 & 0 & 17 & 0.723 & $0.725-$ & 1.000 \\
& (approx:65kg) & 73 & 3 & 70 & & 0.892 & \\
\hline Rearing & $>40$ viss & & & & & & 0.548 \\
system & Backyard & 29 & 0 & 29 & 1.475 & $0.575-$ & 0.773 \\
\hline Feed type & Free-range & 61 & 3 & 58 & & $0.156-$ & $0.015^{*}$ \\
& Raw feed & 23 & 3 & 20 & 9.049 & 0.338 & \\
\hline Presence of & Yeoked feed & 67 & 0 & 67 & & $0.196-$ & $0.025^{*}$ \\
rodents & No & 23 & 3 & 24 & 7.241 & 0.388 & \\
\hline Anthelmintic & Yes & 36 & 0 & 36 & 2.069 & $0.491-$ & 0.272 \\
usage & No & 54 & 3 & 51 & & 0.699 & \\
\hline
\end{tabular}

$*$ Significant association at 0.05 level.

\subsection{Findings of other parasitic infections in muscle samples}

From those examined samples, Cysticercus cellulosae $(6.6 \%, 6 / 90)$ from tongue muscles and Cysticercus tenuicollis $(1.1 \%, 1 / 90)$ from diaphragmatic muscle were also observed.

\section{Discussion}

In the present study, the prevalence of Trichinella was observed as 3.3\%. Therefore, it was higher than the previous local report [8]. It might be due to differences in study place, sample size examined and pig rearing habit. However, the finding of present study was much lower in comparison with that of other countries such as $50 \%$ swine trichinellosis in China and $4 \%$ in Thailand [10].

ICT (International Commission on Trichinellosis) recommended that lowest infection levels was 3-5 lpg (larvae per gram) for proficiency tests, and the generally accepted sensitivity of the artificial digestion method was approximately $3 \mathrm{lpg}$, which is considered sufficient to protect human health [11]. The result of the present study was only $0.2 \mathrm{lpg}$ and so that the level of trichinellosis in domestic pigs in Myanmar tends to the lowest level. There would be due to ingestion of few infective larvae resulting from low level of distribution in Myanmar.

Among the hypothesized risk factors examined, types of feed (use of uncooked feed) and presence of rodents around the pig farm were observed as the factor that significantly associated with trichinellosis. However, Ojodale reported that the associated risk factors in farmed pigs were scavenging pigs, no rodent control, leftovers and dead animals feeding to pigs, interaction with stray pigs, seen snakes around theirs, awareness on trichinellosis and aware of pork-borne diseases except rodents around/within pigsty [12]. According to the questionnaire data, most of the smallholder pig farmers usually collect swill and kitchen leftover from neighbouring houses where might have pork and brown rats eating habits. Feeding of swill and kitchen leftover might favour the presence of Trichinella infected meat scraps in swine feed. From this study, owners of 23 pig 
samples $(25.6 \%)$ did not cook their pig feed containing swill, kitchen leftover. The three positive pigs were from those owners who do not have cooking habit of pig feed.

Various researchers have shown that the cooking of garbage before feeding the pigs is constituted as an important step in the control of human trichinellosis elsewhere [13-17]. Therefore, uncooked swill feeding might serve as a source of infection in pigs and kitchen leftover might contain Trichinella infected meat scraps. Treating such as boiling of feed should be strongly enforced by veterinary public health officials to all pig farmers. Consequently, it could be assumed that the low level of infection observed in this study may be due to the practice of cooking habit in the study pig farms.

Another identified associated risk factor was the presence of rodents and its holes around the pig farms. Twenty seven (30\%) pigs out of 90 showed evidence of high population of rodents around the pig farms with no rodent control and all positive samples $(3.3 \%)$ were collected from those pig farms. Moreover, owner of one positive pig was a retail seller of fried brown rat, wild cat and snake meat in that village and another one positive sample's owner was a pig slaughterhouse worker. Therefore, it could be considered that those positive pigs might have access to synathropic animals (especially rodents) due to presence of rodents around the pig farms and study on the presence of trichinellosis in reservoir hosts should be done in further study. The pig of slaughterhouse worker might also access Trichinella infected meat scraps.

Data on breed (local) of pigs and occurrence (absence) of tail biting is the same response and thus those two factors did not contain in analysis. Other hypothesized risk factors, age, gender, body weight, type of rearing system and usage of anthelmintic, were observed having no association with trichinellosis. Pig farming system was observed as no significant associated risk factor in this study. This may be due to the contact of pigs from backyard farms with synanthropic animals like free-range pigs. In Myanmar, most of the pigs farms are backyards and free range type. According to the finding of this study, both types of pig farming systems were not different in the occurrence of trichinellosis. Therefore, it could be suggested that confinement housing system should be developed in pig industry of Myanmar to efficiently prevent trichinellosis in pigs and also in human.

In this study, examination of trichinellosis was performed by using direct larvae detection method or artificial digestion method (magnetic stirrer method). Today, many methods have been developed for detection of trichinellosis, however, other techniques for detection of trichinellosis such as ELISA, immunofluorescence test and the molecular approach have not been tried yet in Myanmar. Among the various digestion methods, the magnetic stirrer method is the most widely recognized and used, and is recommended by various authorities as the gold standard [12].

According to the findings in this study, swine trichinellosis occurred with a low rate of infection, but farmers should be aware of potential risk factors, especially feeding of raw swill to their pigs.

\section{Conclusion}

Among the total of 270 muscle samples from 90 slaughtered pigs, 3 samples (3.3\%) were observed as positive for trichinellosis by artificial digestion method. Type of feed (use of uncooked feed) and presence of rodents around the pig farms were associated with the occurrence of trichinellosis.

\section{Acknowledgements}

The authors would like to acknowledge slaughterhouse officials and co-operated farmers from Pyinmana, Lewe and Tatkon townships, Nay Pyi Taw, Myanmar. 


\section{References}

[1] K.D. Murrel, B. Fried, Foodborne parasitic zoonoses, first ed. B.J. Samual, S.J. Richard (Eds.), Springer, New York, 2007, pp. 6-7.

[2] World health Organization (WHO), Imported human outbreak of trichinellosis, Italy, Wkly. Epidemiol. Rec. (2001) 97-98.

[3] J. Dupouy-Camet, Trichinellosis a worldwide zoonosis, Vet. Parasitol. 93 (2000) 191-200.

[4] M. Shakespear, Zoonoses, second ed., Pharmaceutical Press, London, 2009.

[5] C.M.O. Kapel, Host diversity and biological characteristics of the Trichinella genotypes and their effect on transmission, Vet. Parasitol. 93 (2000) 263-278.

[6] W.L. Kociecka, Trichinella, in: International handbook of foodborne pathogens, M.D. Miliotis, J.W. Bier (Eds), Marcel Dekker, New York, pp. 634-641.

[7] K.D. Murrel, E. Pozio, Worldwide occurrence and impact of human trichinellosis; 1986-2009, Emerg. Infect. Dis. 17 (2011) 2194-2202.

[8] N.N. Maw, T.T. Kyaw, Z. Aung A, Survey on incidence of trichinosis in pigs in Insein abattoir, Myanmar Vet. J. (2006) 23-25.

[9] M. Thrusfield, Surveys, In: Veterinary Epidemiology, second ed. Butterwords and Co. Ltd., London, 1995, pp. 178-189.

[10] Y. Takahashi, L. Mingyuan, J. Waikagul, Epidemiology of trichinellosis in Asia and Pacific Rim, Vet. Parasitol. 93 (2000) 227-293.

[11] World Organization for Animal Health (OIE) (2008), Trichinellosis, in: Manual of Diagnostic Tests and Vaccines for Terrestrial Animals, Chapter 2.1.16, Available: http://www.oie.int/internationalstandardmanual/access-online/

[12] P.I. Ojodale, V.J. Umoh, I.O. Abdullahi, Detection of Trichinella antibodies in slaughtered pigs and risk factors associated with trichinellosis in pig farms in Kaduna Metropolis, Nigeria, Int. J. of life Sci. and Med. Res. 5 (2015) 1-6.

[13] J.B. McNaught, E.M. Zapata, Incidence of Trichinella spiralis in garbage-fed hogs in San Francisco, J. Exp. Biol. Med. 45 (1940) 701-704.

[14] B.P. Brown, Rodent control and trichinosis, J. Am. Med. Assoc. 117 (1941) 954-954.

[15] W.H. Wright, Public health problems concerned in the disposal of garbage by feeding it to swine, Am. J. Public health. 33 (1943) 208-220.

[16] W.J. Zimmermann, L.H. Schwarte, H.E. Biester, On the occurrence of Trichinella spiralis in pork sausage available in Iowa (1953-60), J. Parasitol. 47 (1961) 429-432.

[17] T.C. Doege et al., Trichinosis and raw bear meat in Thailand. Lancet. 1 (1969) 459-461. 